\title{
Análisis y planificación de Sistemas de Información: tipología y aplicación a la gestión de información
}

\author{
Juan Ros García \\ Departamento de Información y Documentación \\ Universidad de Murcia
}

\subsection{Resumen}

El presente trabajo parte de la Teoría General de Sistemas, propuesta por Bertalanffy. Analiza los distintos modelos de Sistemas de Información y se detiene especialmente en el estudio de la Gestión de la Información , cuyo principal motor es el Information Manager (Autor).

Palabras clave: Gestión de la información. Sistemas de información. Modelos. Information manager.

\subsection{Abstract}

The present work leaves from the General Theory of information systems, proposed by Bertalanffy. It analyzes the different models of information systems and it considers carefully the study of the information management, whose main factor is the Information Manager

Keywords: Information management. Information systems. Models. Information manager.

\section{Introducción (1)}

Los postulados básicos de mi ponencia parten de los estudios de Teoría General de Sistemas de Bertalanffy (1968, 1972, 1975), los estudios de Currás (1988), de González Navarro (1989) y de García Cuadrado (1985). En cuanto a sistemas de información nos atenemos, entre otros, a los estudios de Debons (1988), Senn (1990, 1992), Van Slype (1979, 1988), así como algunos trabajos de López Yepes $(1987,1992)$, de Codina (1994) y trabajos anteriores míos (Ros 1991, 1992, 1993).

La Teoría General de Sistemas supuso una renovación en el concepto de la metodología de la Ciencia. González Navarro (1989) llega a decir que "fue al 


\section{6}

final de la década de los veinte cuando L. Von Bertalanffy sentó las bases de lo que acabaría llamándose teoría general de sistemas (T.G.S.). Al afirmar que, puesto "que el carácter fundamental de un objeto viviente es su organización, el acostumbrado examen de las partes y procesos aislados no puede darnos una explicación completa de los fenómenos vitales", formulaba una idea novedosa en el campo de la investigación biológica -un programa organísmico- que tuvo amplia aceptación. Algún tiempo después -durante los años treinta- formuló de manera oral el concepto de teoría general de sistemas, a la que dio forma escrita después de la segunda guerra mundial. Su propuesta desbordaba ya el ámbito de la biología pues contenía una pretensión de universalidad que no sólo no encontró acogida sino que fue vivamente objetada por muchos. Poco a poco la nueva teoría fue encontrando adeptos, y éstos llegaron a ser tan numerosos que en 1954 se fundó la "Sociedad para el estudio de la teoría general de sistemas", que más tarde se llamó "Sociedad para la investigación en sistemas generales, cambio de denominación que, como se verá más adelante, responde a una razón más profunda que el mero capricho de jugar al hipérbaton”.

El concepto de sistema procede del griego sinestanai, reunir en un todo organizado. Un sistema consiste en lo siguiente:

a) unos elementos que lo componen (subsistemas)

b) una interrelación entre todos y cada uno de ellos

c) un todo distinto de la simple adición, con propiedades suyas, no de los elementos

d) una subordinación de los subsistemas al sistema.

De acuerdo con el concepto de Aristóteles: "el todo es mayor que la suma de las partes" y el todo es un "conjunto de elementos interrelacionados" admitimos una terminología asociada al estudio de sistemas: subsidiaridad, interrelación, retroalimentación, determinismo, teleología, dinamicidad, sinergia y entropía. Sistema de información sería un "sistema incluido en otro sistema más grande, que recibe, almacena, procesa y distribuye información" y cuyos elementos principales serían la información y los procesos de información, admitiendo como Información "cualquier clase de conocimiento o mensaje que puede usarse para mejorar o posibilitar una decisión o una acción".

Según Debons proveen datos, información y conocimientos para resolución de problemas. Dentro del marco de Sistema de Información (S.I.) de Debons, el sistema nos permite ampliar conocimientos, intenta la viabilidad entre el procesamiento de datos (input) y las actividades de búsqueda (output).

Un S.I. convierte los datos brutos en un informe consumible o en un output para otra fase, y según Van Slype, tiene un papel distribuidor, no suplanta la red 
de información de los usuarios, sino que se integra en ella y facilita el flujo de conocimientos.

Existe por ello un problema conceptual. Coll-Vinent (1988) dice que es imposible encontrar una definición, y pese a ello presenta una serie de definiciones, entre las cuales destacaríamos la de Brookes, para quien no deben llamarse S.I. a aquellos sistemas que sólo suministran listas de referencia al usuario que tiene que buscar lo referenciado. Estos S.I. debían llamarse Sistemas de Documentación Automatizada.

Van Slype (1979) distingue entre sistema de información documental y otros sistemas de información. Sobre este tema véanse los trabajos de Codina (1993, 1994), y el trabajo de Tramullas (1995).

Antes de comenzar a dar características y definiciones de los sistemas de información debemos realizar una puntualización. Sabemos que un Sistema de Información no es lo mismo que un Sistema Informático. Además hemos de recordar que no es necesario que un Sistema de Información esté automatizado, si bien no hay ninguno en la actualidad que no lo esté, dado que en estos momentos la mayoría de las bases de datos están automatizadas.

Day y Erdman señalan como características del Sistema de Información (por supuesto informatizado), la potencia del ordenador, la capacidad de almacenamiento, el número de terminales, el software adecuado para lo que del S. de I. se espera. añade nuevos conceptos tales como que atienda a la Organización, la búsqueda de información, y la configuración hardware / software.

Otras definiciones que debemos considerar son las de Debons: conjunto de personas, maquinaria (hardware, equipamiento e instalaciones) y procedimientos que, integrados, hacen posible a los individuos trabajar con inputs (datos, conocimiento) y demandas (decisiones, problemas, que aparecen en el trabajo cotidiano", y "conjunto de personas, máquinas y procedimientos que aumentan el potencial biológico humano para adquirir, procesar y actuar sobre los datos". La de Langefors "Sistema de Información es un sistema incluido en otro sistema más grande, que recibe, almacena, procesa y distribuye información". La de Teichroew, "colección de personas, procedimientos y equipos diseñados, construidos, operados y mantenidos para recoger, registrar, procesar, almacenar, recuperar y visualizar información".

El mismo Coll-Vinent no se sustrae a la tentación de darnos su propia definición: "un conjunto complejo, una serie de cosas o partes conectadas, un cuerpo organizado de cosas materiales e inmateriales". Evidentemente parece más sustanciosa la de Treffel (1985) "conjunto de equipamientos y de logiciales concebidos en vista de una aplicación particular”. Más pragmática es la de Burch (1984), "sistema de información son aquellos subsistemas que manejan

Scire. 2 : 2 (jul.-dic. 1996) 35-52 
todas las actividades del procesamiento de datos, suministran información a una gran variedad de usuarios y pueden ser descritos como diseños y construcción de bloques para demanda". Y como más sencilla y definitiva la definición de Senn (1987) "sistema de Información es un conjunto de personas, datos y procedimientos que funcionan en conjunto".

En otro momento decíamos (Ros, 1991) que definir el Sistema de Información de una organización es una tarea delicada, en particular porque se compone de múltiples procesos que son al mismo tiempo, actores en otros subsistemas de la organización, y porque el sistema de Información participa de toda actividad que se desarrolla en esa organización. A continuación explica el autor que un Sistema de Información sería lo más parecido al sistema térmico de una habitación, que quizá sea difícil de definir y descubrir pero cuyos efectos son palpables.

Sabemos cómo comportarnos, abrir o cerrar ventanas, subir o bajar el termostato, adivinamos que en el interior existe un sistema inteligente y sabemos lo cómodo que es adquirir un servicio llave en mano.

Sabemos también que subyacen determinados oficios: ingenieros, arquitectos, decoradores, obreros, electricistas, calefactores, etc, que han hecho posible tal diseño, tal sistema.

Por tanto "el sistema de información interviene de una forma fundamental en el funcionamiento de una organización. No se puede, pues, definirlo o discutirlo, sin definir o descubrir qué es una organización”.

Hemos dicho que todos los sistemas de información son automatizados. Lo cual comporta una serie de costos en la información final. Al referirse a los costos de la información dice Coll-Vinent (1988) que no serían necesarios los sistemas formales de información, si los managers de una organización pudiesen retener toda la información que necesitan a la hora de decidir; tales sistemas son en rigor una extensión de la memoria, como lo son también los procedimientos ideados por la ciencia para recuperar información.

En la medida que es imposible que la memoria humana, por muy poderosa que sea en algún caso, retenga ni siquiera una pequeña parte de la mucha información que ha de ser constantemente manejada, el sistema de información y las bases de datos que forman parte integrante de él se ha convertido en una herramienta de trabajo imprescindible para el hombre de hoy, más aún para el que ocupa un cargo directivo en un ámbito determinado. Ya pasó el tiempo en que el manejo de la información podía ser considerado como una tarea trivial de recogida, escriben Hartman y otros. Hoy día, añaden, es plenamente reconocida la imposibilidad de controlar cualquier empresa sin unos sistemas de información adecuados. La existencia y el recto funcionamiento de tales sistemas permiten a 
los directores concentrarse en los aspectos de la dirección que son más propios de su misión específica.

Para Van Slype, los elementos que configuran el coste de una cadena son el precio pagado por los documentos, el precio de la mano de obra, los gastos generales y los gastos de material y equipamientos.

A este planteamiento de Van Slype habría que añadir el beneficio económico, si se trata de una cadena documental procedente de un centro de documentación privado, y la previsión de renovación de material y equipamiento si se trata de una cadena de una Organización pública.

El Sistema de Información se enmarca, pues, dentro de la Organización. El S. I. es un conjunto de elementos, ordenadamente relacionados entre sí, de acuerdo con ciertas reglas, que aporta al Sistema Organizacional, es decir a la Organización a la cual sirve, y le marca las directrices de funcionamiento y la información necesaria para el cumplimiento de sus fines. Para llevar a cabo esta tarea, el S.I. tendrá que recoger, procesar y almacenar datos, procedentes tanto de la propia Organización como de fuentes externas, facilitando la recuperación, elaboración y presentación de los mismos.

Las características del Sistema de Información serán:

- tecnológicas, las cuales afectan al rendimiento y a la seguridad del propio sistema.

- funcionales y semánticas, que hacen lo que deben hacer y se adaptan a los cambios necesarios.

- económicas, con unos costos en relación directa con la eficiencia.

- sociales, es decir que tengan en cuenta el entorno en que se mueven.

A la hora de diseñar el sistema de información hay que proceder a un análisis que supone precisar ante todo los objetivos que se propone, mediente un estudio de los usuarios, de los datos de entrada y de los requerimientos al sistema. La planificación debe estar condicionada por el contenido (datos) documental o factual, numérico o textual, por el equipo físico y por los procedimientos.

Sin embargo, cuando diseñemos un sistema de información para una empresa (Coll-Vinent, 1988) no ha de apuntarse tanto a la satisfacción subjetiva tal y como va a ser expresada por el usuario, sino más bien a una mejora de la eficacia del trabajo de resolución de problemas y de toma de decisiones. El usuario -en este caso la organización que encarga el diseño- es el punto de partida de toda reflexión sobre la concepción del sistema: partiendo de las características del usuario han de fijarse las orientaciones principales del sistema.

La definición misma de usuario debe ser considerada como la de management de la organización a la que sirve el sistema que se quiere diseñar. Atender a 
sus necesidades y esas necesidades mismas es lo principal; el éxito de un sistema depende de cómo y hasta qué punto dichas necesidades queden satisfechas.

La dificultad en alcanzar ese éxito es la que explica la tendencia hacia una especie de homologación, consistente en estandarizar los diseños. En vez de que cada empresa se ocupe en diseñar su propio sistema o crearlo ex-novo, para lo cual no cuenta con suficiente número de expertos en su plantilla, compra un sistema básico de procesamiento de datos a los fabricantes de ordenadores o a casas que venden software, cuya función primaria es diseñar, desarrollar u operar procedimientos para procesar datos en sistemas de aplicación universal

Al tratar el análisis y diseño de los sistemas de información, enjuiciando el libro de Senn, decíamos (Ros, 1993) que el autor analiza el diseño de los sistemas de información y se pregunta sobre la conveniencia de un único MIS para la empresa. Afirma que "la experiencia ha demostrado que no funciona el enfoque del sistema total del MIS", y, partiendo de la base de que "un punto de vista mucho más práctico sabe que un sistema ideal no puede ser diseñado", llega a la conclusión de que "un MIS es, por lo tanto, una colección de sistemas de información -un conglomerado de sistemas de información de negocios, relacionados pero independientes, que interactúan unos con otros, si bien operan separadamente. Un MIS podría ser considerado como una "federación" de sistemas o un "supersistema" en el que cada sistema es un bloque integrante de una organización mayor de MIS. Un MIS está compuesto de los Sistemas de Información del área funcional en la organización.Esta estructura permite que los especialistas recolecten la información que mejor conocen". (págs. 462-63). De nuevo parece gravitar sobre Senn la sombra de Toffler en capítulos como la venta mundial (pág. 393), el supermercado inteligente (pág. 133), etc.

\section{Modelos de sistemas de información}

En nuestro trabajo (Ros, 1994) proponíamos los modelos que aparecen en el esquema de la figura1.

El esquema, basado en Debons, abarcaría a nuestro modo de ver todo tipo de sistemas de información. Inicialmente partimos de las ideas de López Yepes (1991) para quien "en relación con las investigaciones que deben producirse para el perfeccionamiento de los sistemas de información creemos que cabe observar tres modelos:

- Modelo A: Sistema contemplado desde una perspectiva general (Individual con subsistemas). Su estudio sirve para el desarrollo del resto de los modelos. Debons lo denomina sistema de información generalizada

- Modelo B : Como subsistema dentro de las organizaciones. De entre ellos podemos destacar dos: 1) Management Information System (MIS) 2) el 
- MODELO A: Sistema que puede ser considerado desde una perspectiva general o individual con los siguientes subsistemas (EATPUT, según las iniciales de cada uno):

\section{Entorno}

2. Adquisición

3. Transmisión

4. Proceso

5. Utilización

6. Transferencia

- MODELO B : Subsistema dentro de las Organizaciones, que puede ser:

1. M.I.S. (Gestión de sistemas de Información)

2. I.M.S. (Sistemas de Gestión de la Información)

- MODELO C : Conjunción de redes y centros de información

Fig. 1. Esquema con los modelos de Sistemas de Información

Information Management System (IMS). Hay numerosas versiones del primero pero,en esencia, se trata de ayudar a las decisiones de la dirección mediante modelos informáticos que facilitan datos, pero por el contrario los IMS facilitan las bases teóricas y técnicas para la gestión de la información en las organizaciones.

- Modelo C: Como resultado de la conjunción de redes y centros de información, enmarcado en las políticas nacionales y territoriales de información. En este sentido el sistema actúa bajo el principio de la centralización, y la red bajo el principio de la coordinación de centros en que, por delegación, se invisten de determinada responsabilidad en la recolección y difusión de fuentes. Desde el sistema, concebido en lo alto de la pirámide, la especialización se va haciendo mayor y todo ellos bajo las directrices de las llamadas políticas de información.

\subsection{Modelo A}

Estaría compuesto por los modelos de Sistema de Información Generalizada que sugieren que los componentes identificados están relacionados con el siste- 
ma. Son descriptivos más que explicativos. No se han visto representando una teoría de SI. Son percepciones de los S.I. y guían su estudio, su análisis y su diseño.

Entre éstos estaría el sistema viviente de Miller (1978), y el modelo (EATPUT) de Debons con seis componentes o subsistemas:

1) Event world (Entorno): Los acontecimientos que son relevantes al objetivo y funcionamiento del S.I. Incluye el proceso, categorización y clasificación de los acontecimientos.

2) Acquisition (Adquisición): Se usa para capturar materia y energía del entorno externo.

3) Transmission (Transmisión): El movimiento actual de los datos dentro de los componentes del sistema.

4) Processing (Proceso): Pedido, almacenaje y recuperación de datos para aplicarlo al problema, resolviéndolo, decidiendo o aplicándolo al desarrollo general.

5) Utilization (Utilización): El componente que representa el requerimiento de evaluación e interpretación de los S.I.

6) Transfer (Transferencia): Es el componente de acción del sistema. La función de transferencia en este modelo se puede ver como comunicación o como transferencia de información.

\subsection{Modelo B}

Está compuesto por los Sistemas de componentes orientados y por los Sistemas de funciones orientadas.

\subsubsection{Sistemas de componentes orientados.}

Además de los SI. de Miller y Debons, hay otros modelos que representan estados en la naturaleza y función de los componentes (subsistemas) del S.I.

Son, por ejemplo el modelo de Yovits, el modelo de Samuelson, el modelo de Shannon (Martínez Montalvo, E.; Martínez Comeche, J.A. 1994), el modelo de Heilprin y el modelo de Otten.

\subsubsection{Sistemas de funciones orientadas}

Los Sistemas de funciones orientadas (F.O.) son sistemas de componentes orientados, tales como gestión, recuperación de datos, diagnóstico. Son competencia del campo de las computadoras. Muy importantes para los científicos de la información y para sus requerimientos interdisciplinares.

- M.I.S. (Gestión de Sistemas de Información). Combinan aspectos de la teoría de la gestión, del proceso de datos, de la teoría de la comunicación. 
Hay numerosas versiones y suministran software suficiente para la gestión.

- I.M.S. (Sistemas de Gestión de la Información)

- D.S.S. (Sistemas de soporte de Decisión): Suministran apoyo para el proceso de toma de decisiones, empezaron como sistemas de decisión de gestión, son semejantes a los MIS, pero los MIS ayudan a resolver problemas estructurados y los DSS ayudan a resolver problemas no estructurados. Están orientados a apoyar problemas creativos. Sus características han sido analizadas por Scott señalando que "se centra en los procesos de decisión, se diseña fácilmente, pues tiene una estructura simple, se realiza y se modifica con rapidez, es diseñado y dirigido por los gestores, suministra información útil para análisis de gestión, se interesa sólo en un área pequeña. Resulta lógico que intente imitar, como el gestor analizaría la situación. Tiene la DB ( bases de Datos) que contiene la información extraída de otros expedientes de la Organización e información del entorno externo. Permite al gestor evaluar los posibles resultados de las distintas alternativas".

- I.A. : Sistemas expertos que ayudan a la toma de decisiones y problemas añadiendo un nuevo elemento, la inteligencia artificial. Los sistemas expertos incluyen una clase profusa de sistemas capaces de llevar a cabo y manipular los símbolos, cuyos componentes son base de conocimiento ( datos reunidos de expertos; relaciones lógicas entre los elementos de los datos),

- Sistemas de gestión de la base del conocimiento: Sistemas de deducción, la lógica en la combinación de datos de los expertos hasta conseguir intuiciones o soluciones a los problemas o requerimientos de decisión.

\subsection{Modelo C}

Consisten en personas, medios y tecnología que suministran información y conocimiento y que constituyen parte de su trabajo, su medio de vida. Hay dos modelos principales:

\subsubsection{Modelo de Havelock}

El modelo de Havelock, ha sido descrito por Debons (1988). En él vemos que los sistemas de información que consisten en personas, tecnología y procedimientos que ayudan a quienes necesitan información y/o conocimiento como parte de su trabajo o profesión, o, justamente para ganarse la vida. A veces no está claro si los autores de estos conceptos consideran el sistema como el cuerpo de la información o un algo que capacita a que alguien obtenga el cuerpo de la información o el conocimiento. Podemos defender que todas estas hipótesis son posibles. Es importante reconocer, sin embargo, que pueden estar envueltos diferentes procesos al tratar las diferencias que están implicadas.

Havelock, un psicólogo social interesado en el uso del conocimiento entre los Scire. 2 : 2 (jul.-dic. 1996) 35-52 
científicos, examinó las formas en las que éstos y otros profesionales obtienen información científica y técnica, y conocimiento como parte de su trabajo. Consideró que la información era una necesidad fisiológica y, por tanto, propuso un modelo de entorno, en forma de clínica que pudiera satisfacer esta necesidad.

La descripción de Havelock de la clínica incluye instituciones afines (bibliotecas, centros de información, etc) y procedimientos que enlazan los puntos integrantes en un sistema social de utilización de conocimientos comprensivos.

\subsubsection{Modelo de Taylor}

El modelo de Taylor es un sistema de información que hace referencia a entornos de gentes, tecnologías y procedimientos que incluyen las características siguientes:

- Son procesos, formalmente diseñados, que seleccionan / adquieren / organizan / archivan / recuperan / exhiben / analizan y/o interpretan mensajes.

- Son procesos por donde los mensajes son realizados por señales o por alteración del contenido

- Los mensajes son comunicaciones finales: se publican, se graban, y se presentan en una memoria.

- Los mensajes están también generados a través de artificios y procesos.

- Estos mensajes son buscados por otros o suministrados automáticamente a otros.

De la conjunción de los sistemas, redes y centros de información, y de las políticas de información surge este tercer tipo de Sistemas de Información. Sólo el plan de estudios de la Diplomatura de Murcia y de la Universidad Complutense de Madrid recogen esta conjunción que también han incluídoen sus proyectos docentes las profesoras Ubieto y García Cuadrado.

\section{Los sistemas MIS}

Detengámonos un poco en los sistemas MIS. Recordemos que según Senn (1992) un Sistema de Información es "un conjunto de personas, datos y procedimientos que funcionan en conjunto".

El "énfasis en sistemas significa que los variados componentes buscan un objetivo común para apoyar las actividades de la organización. Estas incluyen las operaciones diarias de la empresa, la comunicación de los datos e informes, la administración de las actividades y la toma de decisiones".

Las organizaciones tienen sistemas de información en el que se reflejan las compras, ventas, cobros, pagos, ganancias, pérdidas, etc. El sistema ayuda a las 
decisiones: comprar, almacenar, vender, esperar, rebajar, etc.

El S.I. no tiene por qué ser automatizado, ya que "si un sistema del tipo normal de procedimientos y personas puede hacer un trabajo eficientemente y sin error, habrá pocos motivos para utilizar computadoras. Sin embargo, a menudo, cuando crece el volumen de trabajo los procedimientos aumentan en complejidad, o las actividades llegan a estar más interrelacionadas, obteniéndose grandes mejoras al introducir la ayuda de un sistema de cómputo". Con todo, tal como decíamos arriba "no siendo necesario ni imprescindible que sea informatizado, no existe ninguno que no lo sea".

Las funciones del sistema de información son: recibir datos (información), actuar sobre ellos y generar información, y dar salida de la información al usuario (que puede ser un gerente, un administrador o un directivo).

Piénsese, por ejemplo, en el tipo de sistema de información que procesa en un banco la información relativa al solicitante de un préstamo y la entrecruza con la información interna de los fondos, volumen de riesgo, etc, del banco en ese momento. Esta información se ofrece al directivo que tiene que tomar una decisión.

Igualmente ocurre con sistemas de información similares en la aviación civil: boletos, equipaje, carga, asientos, tripulación, etc.

La información es considerada, pues, como un instrumento, y, los sistemas de información se configuran como " medio de mejorar la actividad empresarial y, especialmente, al servicio de la toma de decisiones propiciando en la medida de lo posible la canalización de los documentos constitutivos de las comunicaciones internas y externas. La función documental en la empresa atendería, desde estos presupuestos, a dos grandes líneas de orientación: la especialización de la misma y la organización de los sistemas de documentación empresarial.

- Los sistemas de procesamiento de transacciones procesan los datos referentes a las actividades de la empresa -por ejemplo, ventas, colocación de pedidos y movimiento de almacén e inventario. La función en sí, que se llama procesamiento de transacciones se funda en la operación metódica de cualquier empresa u organización. Las cinco razones para el procesado de las transacciones son la clasificación, cálculo, distribución, resumen y almacenamiento de los datos.

- Los sistemas de información gerencial, llamados también sistemas de soporte de gerencia se enfocan al apoyo para la toma de decisiones cuando los requerimientos de información pueden ser identificados de antemano. En otras palabras, la información que un administrador o un usuario final

necesita puede ser determinada después de un análisis minucioso de la

Scire. 2 : 2 (jul.-dic. 1996) 35-52 
situación.

- Los sistemas de apoyo para la decisión son sistemas de información cuyo propósito es auxiliar a los administradores con las decisiones únicas que no se repiten y que carecen relativamente de estructura. Una decisión para el establecimiento de precios está relativamente estructurada: es evidente qué información es necesaria y tal información por lo general ya existe.

- Los sistemas de información para oficinas son una clase especial de un sistema de procesamiento de información que puede usarse en ellas. Los sistemas para oficinas integran aspectos de los sistemas de información descritos anteriormente, así como el procesamiento de palabras y la teletransmisión de datos.

Los sistemas de información, según acabamos de ver, se clasifican en cuatro clases diferentes, pero relacionadas entre sí: sistemas de procesamiento de transacciones, de información para la gerencia, de apoyo para la decisión y de información para oficinas. Cada modalidad de sistema desempeña un importante papel en el control y la coordinación de muchas partes o componentes de una organización o empresa.

Los sistemas de computación y el procesamiento de datos basado en computadoras han alterado en forma significativa la capacidad de las empresas para procesar transacciones y tomar decisiones. Los diferentes tipos de sistemas incluyen macrocomputadoras, minicomputadoras y maxicomputadoras. Sin tomar en consideración su tamaño, las computadoras están controladas por conjuntos de instrucciones denominados programas. Los programas señalan a la unidad central de procesamiento cómo operar con los datos de entrada, cómo procesarlos y a dónde y cómo enviar o manifestar los resultados de salida. Como se verá los sistemas de computación son una parte integral de los sistemas de información. No obstante, la computadora por sí sola ocupa un lugar secundario respecto de la información que brinda a los administradores y a otros usuarios.

Lógicamente, Senn parte, para el análisis de los sistemas de Información, del concepto de Información. Cabe recordar lo dicho por el Profesor López Yepes, considerando la información como a) bien económico, b) como proceso, modo y medio informativo, c) como mensaje informativo, d) como proceso documental ,y e) como elemento del proceso del conocimiento. Senn analiza muy someramente la "explosión" de la información, para definir la información gerencial o administrativa como "conjunto de datos que se presentan de forma que es inteligible al receptor ". La carencia de información produce incertidumbre, y deben proporcionar una información de apoyo, de avance, de advertencia, de planeación, de operaciones internas, confidencial, e información difundida al exterior.

Hasta aquí hemos visto la información como instrumento al servicio de las

Scire. 2 : 2 (jul.-dic. 1996) 35-52 
organizaciones, si bien podríamos profundizar en otros muchos aspectos, sociológico, técnico y económico.

De la concepción de información como servicio se pasa a la de recurso económico. "Sin duda, la razón última del nuevo giro en la cuestión objeto de estudio es el paso de la concepción de información como servicio a la de recurso económico, a la de bien informativo. En efecto la información es un bien, bien capaz de generar un valor determinado cuando aumenta su papel en aquello que puede afectar a la empresa y cuando incide en la acertada toma de decisiones. La importancia de esta consideración va creciendo en España considerablemente" (Ros, 1993).

Como dice Joan Majó: "pero todas ellas encubren un hecho fundamental: ha aparecido un nuevo factor de producción, la información y su utilización permite con ventaja la sustitución de los factores clásicos. Una mejor tecnología de proceso (información sobre "cómo hacer las cosas") permite ahorrar energía y materia primas. Una mejor logística (información sobre cómo combinar las cosas) permite utilizar menos capital. Una adecuada automatización (informática) sustituye mano de obra".

A medida que se va introduciendo más información, e información más estructurada, en el proceso de producción se van consiguiendo mejores condiciones de competitividad y se van sustituyendo los otros factores de producción.

La rapidez con la que una sociedad se va adaptando a esta nueva situación es la clave de su éxito económico. El futuro desarrollo no depende de disponer de mayor cantidad de recursos energéticos, minerales o incluso financieros. El futuro depende de disponer de información y de la tecnología suficientes. Todo ello conduce, tanto en lo individual como en lo colectivo, a una clara prioridad social: la educación, la formación, el incremento del nivel de conocimiento y de la capacidad para adquirirlos, de todos y cada uno de los miembros de la sociedad".

Se mezclan, en las Organizaciones los MIS, se complementan, se perfeccionan; y generan, a su vez, Información.De ahí la necesidad de gestionar esa información, y la necesidad del gestor de información, entre otros.

\section{Los I.M.S.}

Damos por supuesto todo lo relativo al nacimiento de IM (Information Management), pero para lo que nos ocupa seguimos López Hernández (1991):

"Nacieron los sistemas de información en las organizaciones, cuyo fin no era simplemente mejorar un trabajo rutinario, sino servir de apoyo a la gestión de todas las actividades de la empresa desde la perspectiva de la dirección. En la década de los 70 surgieron y se perfeccionaron instrumentos con base TI para llevar a cabo la tarea de toma de decisiones de manera centralizada: los DSS (Decision Support Systems) y los 
MIS (Management Information Systems). Estos últimos se definen como "sistemas que recuperan los datos del entorno, que capturan los datos de las transacciones y operaciones que tienen lugar en la empresa y que filtran, organizan y seleccionan los datos y los presentan como información para los responsables".

Así es como se ha dado un paso gigantesco en la concepción actual de la empresa como un organismo en el que juega un papel clave el fenómeno informativo.

Al principio de la década de los 80 , siguiendo el camino abierto en los EE.UU., se ha avanzado en Europa hacia un nuevo concepto, que es el que tratamos de exponer aquí: la Gestión de la Información (Information Management) como eje central de la dirección y administración de las empresas. De la creación de sistemas de información en las organizaciones (SIO) para centralizar y procesar todos los datos necesarios para el funcionamiento organizativo y ayudar en la toma de decisiones se está pasando progresivamente a concebir las organizaciones mismas como sistemas informativos (OSI), elevando así la información y el conocimiento al primer plano en todos los ámbitos de la actividad organizacional; considerando la empresa esencialmente como un sistema de información, entre otras razones por tratarse de un conjunto compuesto de individuos entre los cuales se establecen lazos y redes de comunicación, sobre cuya base el sistema entero actúa coordinadamente para mejor alcanzar los objetivos globales".

Las empresas sienten la necesidad de introducir innovaciones para sobrevivir. I.M. es una innovación que facilita todas las innovaciones. Organizar la empresa como S.I. es no sólo introducir innovación sino estar en estado continuo de innovación.

Los IMS (Lopez Yepes, 1991) facilitan las tareas teóricos y técnicas para la gestión de la información en las organizaciones. Constituyen el objeto de una disciplina muy extendida en Gran Bretaña, Information Management, que trata de identificarse plenamente con Information Science y que parte de consideraciones como: la información es un bien económico, la información es el nervio de la organización y la organización es en sí misma un sistema de información. Un autor español decía recientemente que la consideración de la información como recurso clave de las organizaciones conduce a una visión global de la misma y a asignarle en la empresa el papel directivo que le corresponde... Un cambio estructural que permite en las organizaciones adaptarse con más eficacia al medio ambiente es precisamente la introducción de la figura del information manager. Porque lo que implica este cambio es la reestructuración de todo el sistema nervioso (informativo) de la empresa para percibir mejor las variaciones del medio y darse así las respuestas más adecuadas en cada momento” 


\section{El Information Manager}

No hemos de tener ninguna duda de que el cambio fundamental es introducir el information manager. Sin embargo, surgen dudas.

"Pero (Ros, 1993) ¿cuáles son las notas características de este Information Manager? ¿Dónde se forma? ¿Cómo adquiere sus conocimientos? ¿Están fijadas sus competencias y sus misiones en las Organizaciones? ¿Son iguales sus cometidos en distintas organizaciones?

He aquí algunas cuestiones que hoy nos ocupan y nos preocupan. Horton ha señalado las dificultades de definir nítidamente los sujetos de los procesos de tratamiento y gestión de la información. Junto a los usuarios, cita a los Information Suppliers, que crean o producen productos y servicios de información a los usuarios. Se sitúan entre éstos y los Information Handlers que transforman los datos en información para su utilización (estadísticos, bibliotecarios, information brokers). El Information Counselor, aparece entre el usuario y el Information Handler o entre el usuario y el Information Supplier. Finalmente, la figura del Information Manager".

Hoy pensamos que el Information Manager, el gestor de información debe asumir las tareas de la dirección de la tecnología informativa en los negocios, la política de gestión y transformación de la tecnología informativa, la planificación de bases de datos, software, inovaciones técnicas, etc, la gestión de los recursos humanos y técnicos. Ha de ser un especialista en planificar, coordinar y controlar recursos y debe operar en las áreas de recursos informativos (con funciones de identificación, valoración y uso), en el área de conocimiento de la tecnología necesaria para recoger y almacenar la información, y para procesarla, recuperarla y distribuirla, y, finalmente, ha de operar en el área de gestión, atendiendo a la planificación general, a los recursos humanos, a la comunicación interpersonal, a la contabilidad y al marketing.

En otras palabras el gestor de información debe organizar el IMS. Cronin (1985) para quien "Information Management es un aspecto integral en la gestión de las organizaciones. Es una respuesta articulada para el desarrollo de factores interrelacionados, como los siguientes: a) el continuo crecimiento del volumen y variedad de la información documentada; b) la convergencia de las tecnologías asociadas a la creación, comunicación y diseminación de la información; c) la importancia cada vez mayor de la información como recurso clave de las organizaciones; d) la necesidad de gestionar este nuevo recurso de modo más eficaz", habla de la recionalidad sinóptica.

Linda Woodman (1985) que ha dado una de las definiciones nás sugestivas "Gestión de la información es todo lo que se refiere a conseguir la información adecuada, para la persona adecuada, al coste adecuado, en el momento adecuado,

Scire. 2 : 2 (jul.-dic. 1996) 35-52 
en el lugar adecuado, para tomar la decisión adecuada", dice que la planificación del IMS tropieza con obstáculos tales como la limitación del software, la inmediatez de las necesidades de información, la sobrecarga de información y la diversidad de lenguajes.

Con todo los obstáculos pueden solucionarse: Keen propone un mapa de interconexión de medios informativos, David Best propone la cartografía informativa y Flood insiste en aspectos de la organización y del staff y en la automatización (procesos de datos, bases de datos, microsistemas, sistemas automáticos de oficinas) y termina considerando fundamental el perfeccionamiento del sistema del IM (redes de comunicación, distribución de la información, localización de la información, índice de la información, memoria de la empresa).

Holloway (Ros, 1993) señala estas posibilidades a la hora de enumerar los útiles y técnicas para desarrollar los IMS:

-D. M. S. (Database Management Systems): Permiten el acceso a los datos

-D. D. S. (Data Dictionary Systems): Que permiten el control automático y la documentación de los datos.

-Q. L. (Query Languages): Que permiten al usuario final el acceso directo a la búsqueda de datos.

-4th Generation Systems: Permiten planificar y controlar el desarrollo y evolución de los sistemas de evaluación.

Con todo, el tema del gestor de información está empezando. Las funciones señaladas por Holloway son funciones plurales que hablan de la pluralidad funcional del gestor de información. O quizá de varias funciones o varios oficios con varias funciones.

\section{Notas}

(1) Quiero expresar, antes de nada, mi agradecimiento a la universidad de Zaragoza y concretamente a la organización de IBERSID'96 por la oportunidad de estar nuevamente aquí y contribuir, modestamente, a este encuentro sobre "Sistemas de Información y Documentación: la planificación y gestión de sistemas de información". Si bien he de destacar que mi ponencia, más que en planificación y gestión de sistemas de información (que serían los MIS) se centra en la aplicación de estos sistemas a la gestión de la información (que serían los IMS).

Por otra parte, la ubicación de mi ponencia tras la intervención de la doctora Currás y su, sin duda, esclarecedora visión de la Teoría General de Sistemas, y tras la del Dr. Codina, referente obligado en la tipología de sistemas, así como tras la intervención del Dr. López Yepes sobre la utilización de estos sistemas en los Centros de Documentación, me evitan tener que teorizar excesivamente sobre algunos de estos conceptos por considerarlos adecuadamente explicados. 


\section{Bibliografía}

Bertalanffy, L. von. (1968) Teoría General de Los Sistemas. México : Fondo de Cultura Económico, 1968.

Bertalanffy, L.von. (1972) Historia y situación de la teoría general de sistemas. // Tendencias en la Teoría General de Sistemas. Madrid : Alianza Universidad, 1972. 29-53.

Bertalanffy, L. von. (1975). Perpectivas en la Teoría General de los Sistemas. // Estudios científico-filosóficos. Madrid : Alianza, 1975.

Codina, Ll. (1994). La naturaleza de la recuperación de información : implicaciones para el diseño de Sistemas de Información Documentales. // Documat 94. Gijón : 1994. 433 ss.

Codina, L1. (1994). Modelo conceptual de un Sistema de Información Documental. // Revista española de Documentación Científica. 17: 4 (1994) 440 y ss.

Coll Vinent, R. (1988). Información y poder : El futuro de las bases de datos documentales. Barcelona : Herder, 1988.

Cronin, B.(Ed.).(1985) Information Management : From Strategies to Action. London : ASLIB, 1985.

Currás, E. (1988) La información en sus nuevos aspectos : ciencias de la documentación. Madrid : Paraninfo, 1988.

Debons, A. y Larson, A. (Eds.) (1983) Information Sciences in Action. Boston : Martinus Nijhoff Publishers, 1983.

Debons, A; Horne, E. y Cronenweth, S.(1988). Information Science : An integrated view. Boston : G.K. Hall and Co., 1988.

García Cuadrado, A. (1995). Notas sobre la Teoría General de Sistema. // Revista General de Información y Documentación . 5: 1 (1995)

González Navarro, F. (1989). La Teoría General de Sistemas como matriz disciplinar y como método jurídico. // Persona y Derecho. 21 (1989). 92-149.

Langefors, B.(1976). Teoría de Sistemas de Información. Buenos Aires : El Ateneo, 1976

López Hernandez, J.(1990). La Gestión de Información en las Organizaciones : una disciplina emergente. // Documentación de las Ciencias de la Información. 13 (1990) 133148.

López hernández, José (1991). Tres lecciones de Documentación General. // Cuadernos E.U.B.D. Complutense. 1 : 2 (1991)

López Yepes, J.(1991). Desarrollo de los sistemas de Información y Documentación // Cuadernos E.U.B.D. Complutense. 1: 2 (1991) 23-33.

López Yepes, J.(1987). Introducción a Los Sistemas de Información en las Organizaciones. .// Cuadernos de Documentación de Cajas de Ahorros. 3 (1987) 5-6.

Martínez Montalvo,E.; Martínez Comeche, J. A. (1993). Adecuación de modelos matemáticos a la Ciencia de la Documentación. // Documentción de las ciencias de la Información. 16 (1993) 155-168.

Ros Garcia, J. (1991). El reto de la moderna organización. // Cuadernos E.U.D.B. Complutense. 1 : 2 (1991) 35-50.

Scire. 2 : 2 (jul.-dic. 1996) 35-52 
Ros Garcia, J. (1993). Auge de los sistemas de información y Documentación en las Organizaciones. // Cuadernos de Documentación Multimedia. 2 (1993).

Ros Garcia, J. (1992). Gestión de la Información y la Documentación en las organizaciones (Tipología y funciones del Information Manager). Seminario Hispano-Cubano, Madrid. // Revista General de Información y Documentación. 3:1 (1992).

Ros Garcia, J.(1992). Sistemas de información. Tendencias, evolución y repercusiones. // Ciencias de la Información, 23:2 (1992) 83-96.

Ros Garcia, J., Fernandez, M.;Aguilar, S.(1992). Gestión de la Información y documentación en las organizaciones : una aportación bibliográfica.// Revista General de Información y Documentación. 2 : 1 (1992) 33-70.

Senn, J.A.(1992). Análisis y diseño de sistemas de información. 2ed. México, etc. : Mc Graw-Hill, 1992.

Slype, G. van. (1979). Conceptión et Gestion des Systèmes documentaires. Les editions d'organisation : Paris, 1979.

Slype, G. van. (1988). Documentologie. 2 Vol. Bruxelles: Press Universitaires de Bruxelles, 1988.

Slype, G.van. (1991). Los lenguajes de indización: concepción, construcción y utilización en los sistemas documentales. Trad. de Pedro Hípola y Félix de Moya. Madrid, Salamanca : Fundación Germán Sánchez Ruipérez, 1991.

Sotolongo Aguilar, G.R.(1993) Derroteros de la Gestión de Información y Documentación en las organizaciones. Seminario Hispano-Cubano, Madrid, 1992. //Revista general de Información y Documentación. 3 : 1 (1993).

Tramullas, J.(1995). Sistemas de Información para la Arqueología.. [Tesis doctoral]. (inédita) Zaragoza 1995, 2 vol.

Woodman, L.(1985). Information management in large organisation // Cronin, B.(ed.). Information Management :. From strategies to action. London: ASLIB, 1985. 